\title{
Network Structures and Dynamics Of Early Dementia Events Recorded in Primary Care Electronic Health Records
}

\author{
Maxine Mackintosh \\ Institute of Health Informatics \\ University College London \& Alan Turing Institute \\ London, United Kingdom \\ maxine.mackintosh.11@ucl.ac.uk \\ Martin Rossor \\ Dementia Research Centre, Institute of Neurology \\ University College London, \\ London, United Kingdom \\ m.rossor@ucl.ac.uk \\ Kenan Direk \\ Institute of Health Informatics \\ University College London \\ London, United Kingdom \\ k.direk@ucl.ac.uk
}

\author{
Arturo Gonzalez-Izquierdo \\ Institute of Health Informatics \\ University College London \\ London, United Kingdom \\ arturo.gonzalez-izquierdo@ucl.ac.uk
}

\author{
Kirstie Whitaker \\ Alan Turing Institute \& University of Cambridge \\ London, United Kingdom \\ kwhitaker@turing.ac.uk
}

\author{
Spiros Denaxas \\ Institute of Health Informatics \\ University College London \\ London, United Kingdom \\ s.denaxas@ucl.ac.uk
}

\begin{abstract}
Background: Identifying early signs of heterogeneous conditions like dementia is challenging. We used electronic health records (EHR) and data-driven methods in order to represent prodromal dementia as a dynamic temporal network of healthcare events and move beyond reductionist representations of complex syndromes.
\end{abstract}

Methods: We used UK primary care EHR data from Clinical Practice Research Datalink (CPRD) through the CALIBER resource and identified patients with a dementia diagnosis. We constructed a weighted, undirected network. We calculated centrality measures of the network and compared three community detection algorithms, Louvain, InfoMAP and Walktrap. Distinctive temporal communities of events in two year windows were derived, and we explored community membership, interactions and dynamics using evaluation measures including Jaccard distance, modularity, Rand Index and Normalised Mutual Information.

Results: We analysed data from 89,102 patients, where nodes $(n=816)$ were connected to edges $(n=392,345)$ based on the frequency with which two features were recorded in the same time window. Across the whole prodrome, repeated cardiovascular medications accounted for $22 \%$ of the network

Permission to make digital or hard copies of part or all of this work for personal or classroom use is granted without fee provided that copies are not made or distributed for profit or commercial advantage and that copies bear this notice and the full citation on the first page. Copyrights for third-party components of this work must be honored. For all other uses, contact the Owner/Author

DPH' 19, November 20-23, 2019, Marseille, France

(C) 2019 Copyright is held by the owner/author(s).

ACM ISBN 978-1-4503-7208-4/19/11.

https://doi.org/10.1145/3357729.3357757 highest eigen centrality (influence on the entire network structure) and QRISK2 and malignant neoplasms had the greatest betweenness centrality (bridge between events). Louvin had the highest modularity and clustered the temporal network into six communities: The largest community was enriched for respiratory diseases (late-prodrome) and circulatory conditions (mid). Community 2 was enriched for musculoskeletal conditions (late), and Community 3 for administrative events (early) and nervous system conditions (late). In the early prodrome, there were more transitions between communities, however from 10 years to diagnosis, most events occurred within communities.

Conclusion: By understanding the interdependencies of conditions and associated medications across a disease network, we can deepen our understanding of prodromal dementia and create an accurate phenotype of the earliest stages of cognitive decline.

\section{CSS CONCEPTS}

Applied computing $\sim$ Biological networks - Applied computing $\sim$ Health care information systems - Applied computing Health informatics • Computing methodologies Cluster analysis • Computing methodologies $\sim$ Principal component analysis

\section{KEYWORDS}

Dementia; Networks; Communities; Electronic Health Records; Clinical Practice Research Datalink; Primary Care

\section{ACKNOWLEDGMENTS}

Research was predominantly funded by a UCL Provost's Strategic Development Fund Fellowship and the Alan Turing Institute 